\title{
Shedding Light on Cadherin Function in Epidermal Sheet Formation and Maintenance of Tissue Integrity
}

\author{
H. Amalia Pasolli, Christopher L. Tinkle, Nicole Stokes, and Elaine Fuchs
}

Howard Hughes Medical Institute, Laboratory of Mammalian Cell Biology and Development, The Rockefeller University, 1230 York Avenue, New York, NY 10065.

Classical cadherins function in intercellular adhesion, polarization and differentiation. The prototype is E-cadherin that establishes adherens junctions between cells by coupling to catenins and the actin cytoskeleton. There is a well-known connection between cadherin/catenin mutations and cancers. However, ascertaining classical cadherin function(s) in epidermis has been complicated by co-expression and gene linkage. Here, we have overcome these difficulties by combining gene ablation/transgenic RNA interference to reveal new roles for E- and P-cadherins in epidermal sheet formation in vitro and maintenance of epidermal integrity in vivo.

By devising skin-specific RNAi technology, we demonstrate that cadherin inhibition in vivo impairs junction formation and intercellular adhesion and increases apoptosis. These defects compromise epidermal barrier function and tissue integrity. Ultrastructural analysis is a valuable tool to assess these alterations. In vitro, with only E-cadherin missing, epidermal sheet formation is delayed, but when both cadherins are suppressed, defects extends to adherens junctions, desmosomes, tight junctions and cortical actin dynamics. Using different rescue strategies, we show that cadherin level rather than subtype is critical.

Finally, by comparing conditional loss-of-function studies of epidermal catenins and cadherins, we dissect cadherin-dependent and independent roles of adherens junction components in tissue physiology. These observations are interesting taking into account the many cancers involving alterations in the expression of both cadherins and catenins and suggest that the loss of one may not functionally equate the loss of the other.

[1] Tinkle CL, Pasolli HA, Stokes N, Fuchs E. New insights into cadherin function in epidermal sheet formation and maintenance of tissue integrity. Proc Natl Acad Sci U S A. 2008 Oct 7;105(40):15405-10.

[2] Tinkle CL, Lechler T, Pasolli HA, Fuchs E. Conditional targeting of E-cadherin in skin: insights into hyperproliferative and degenerative responses. Proc Natl Acad Sci U S A. 2004 Jan 13;101(2):552-7. 

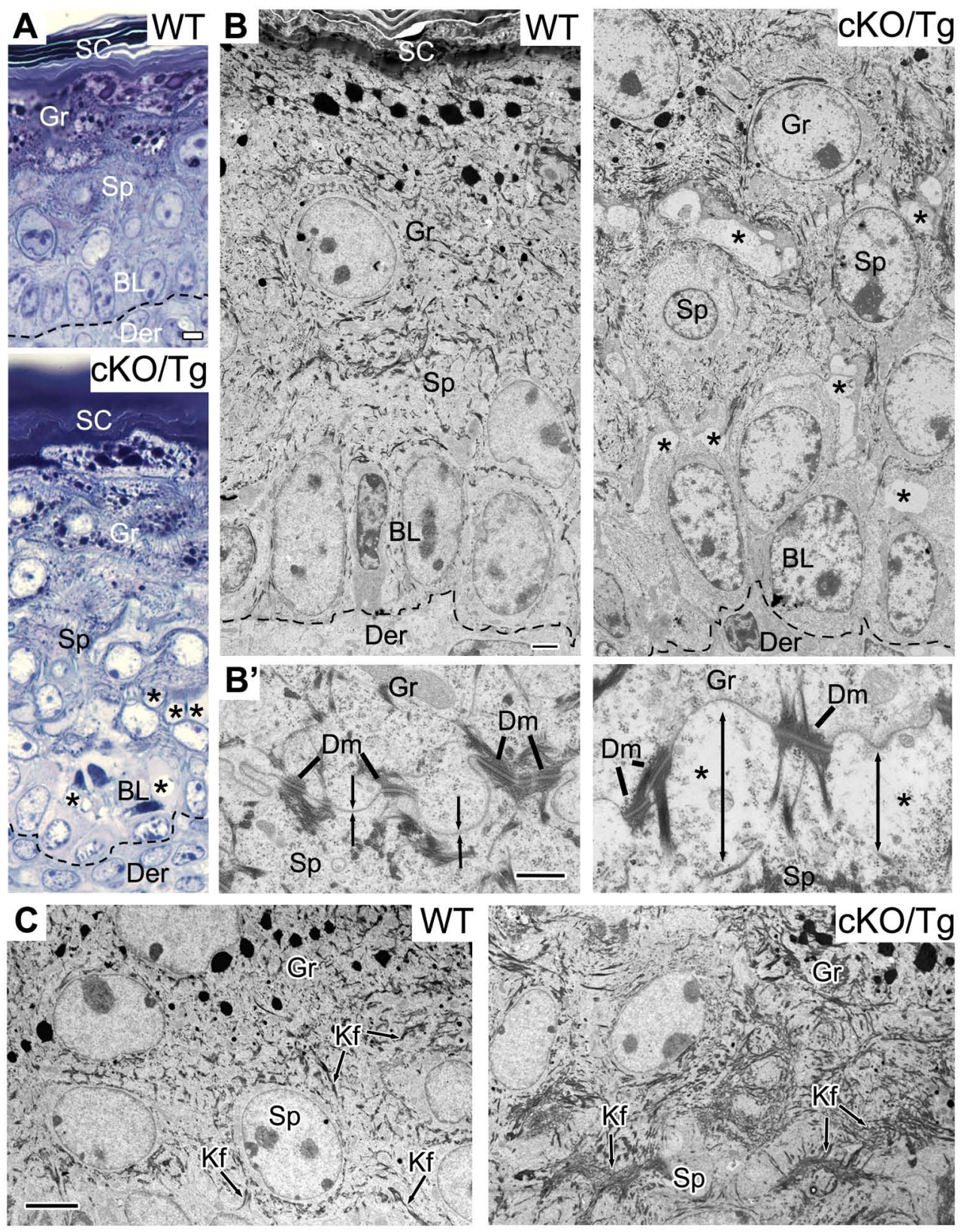

Ultrastructural abnormalities in cadherin-deficient epidermis. (A) 0.8

$\mu \mathrm{m}$ semithin sections of $\mathrm{P} 0$ backskins were stained with toluidine blue. Asterisks denote intercellular gaps between keratinocytes of cadherin-deficient ( $\mathrm{cKO} / \mathrm{Tg}$ ) epidermis. Also note epidermal hyperthickening and marked disorganization and altered cuboidal morphology of basal cells in $\mathrm{cKO} / \mathrm{Tg}$ skin. ( $\mathrm{B}$ and $\mathrm{C}$ ) Transmission electron microscopy. Asterisks in (B and B') and double arrows in (B') denote intercellular gaps. Note that desmosomes (Dm), appear to be intact even in areas where intercellular gaps occurred (B'). Region in C contrasts the normal desmosome-keratin filament network of suprabasal cells of WT epidermis versus the irregular aggregates of keratin filaments (Kf) that were frequently observed in $\mathrm{cKO} / \mathrm{Tg}$ epidermis. Such alterations in keratin organization frequently reflect defects in mechanical integrity. Additional abbreviations: BL (basal layer); Sp (spinous layer); Gr (granular layer); SC (stratum corneum). Dotted lines in A and B represent junction between epidermis and dermis. (Scale bars: A, $5 \mu \mathrm{m}$ (A); B, $2 \mu \mathrm{m}$; B', 500 $\mathrm{nm} ; \mathrm{C}, 5 \mu \mathrm{m}$.) Reprinted from reference [2]. 